\title{
GENERAL FADING DISTRIBUTIONS
}

\author{
Michel Daoud Yacoub
}

\begin{abstract}
This paper presents two general fading distributions - the $\kappa-\mu$ Distribution and the $\eta-\mu$ Distribution. The $\kappa-\mu$ Distribution includes the Rice and the Nakagami$\mathrm{m}$ distributions as special cases. The $\eta-\mu$ Distribution includes the Hoyt and the Nakagami-m distributions as special cases. Therefore, in both fading distributions, the One-Sided Gaussian and the Rayleigh distributions also constitute special cases and the Lognormal distribution may be well-approximated. Preliminary results show that these new distributions provide a very good fitting to experimental data.
\end{abstract}

Keywords: fading distributions, Nakagami distribution, Rayleigh distribution, Rice distribution, One-sided Gaussian distribution, Hoyt distribution, Lognormal distribution.

Resumo - Este artigo apresenta duas distribuiçōes gerais de desvanecimento - a Distribuição $\kappa-\mu$ e a Distribuição $\eta-\mu$. A distribuição $\kappa-\mu$ engloba as distribuições de Rice e Nakagami-m como casos especiais. A distribuição $\eta-\mu$ engloba as distribuições de Hoyt e Nakagami-m camo casos especiais. Conseqüentemente, em ambas as distribuições de desvanecimento, as distribuições de Rayleigh e SemiGaussiana Positiva também são obtidas como casos especiais e a distribuição Log-normal pode ser obtida de forma aproximada. Resultados preliminares mostram que estas novas distribuições proporcionam um bom ajuste com dados experimentais.

Palavras-Chave: distribuições de desvanecimento, distribuição de Nakagami, distribuição de Rayleigh, Rice distribution, distribuição Semi-Gaussiana Positiva, distribuição de Hoyt, distribuição Lognormal.

\section{INTRODUCTION}

The propagation of energy in a mobile radio environment is characterized by mcident waves interacting with surface irregularities via diffraction, scattering, reflection, and absorption. The interaction of the wave with the physical structures generates a continuous distribution of partial waves [1], with these waves showing amplitudes and phases varying according to the physical properties of the surface. The propagated signal then reaches the receiver through multiple paths. If the waves are not resolvable within the available bandwidth or if an appropriate signal treatment is not carried out, the result is a combined signal that fades

Michel Daoud Yacoub is at the Department of Communications of the School of Electrical and Computer Engineering, UNICAMP; DECOM/FEEC/UNICAMP, C.P. 6101, 13085-970, Campinas, SP, Brasil. E-mail: michel@decom.fee.unicamp.br.

Review coordinated by Antonio Sérgio Bezerra Sombra, Ricardo Menezes Campello de Souza, and Max Gerken. Submitted: Dez. 31, 2001, revised: Mar. 15, 2002, accepted: Apr. 8, 2002. rapidly, characterizing the short term fading. For surfaces assumed to be of the Gaussian random rough type, universal statistical laws can be derived in a parameterized form [1].

A great number of distributions exist that well describe the statistics of the mobile radio signal. Extensive field trials have been used to validate these distributions and the results show a very good agreement between measurements and theoretical formulas. The long term signal variation is well characterized by the Lognormal distribution whereas the short term signal variation is described by several other distributions such as Rayleigh, Rice, Nakagami-m, and Weibull, though to the latter, originally derived for reliability study purposes, little attention has been paid. It is generally accepted that the path strength at any delay is characterized by the short term distributions over a spatial dimension of a few hundred wavelengths, and by the Lognormal distribution over areas whose dimension is mucl larger [2]. Three other distributions attempt to describe the transition from the local distribution to the global distribution of the path strength, thus combining both fast and slow fading. These composite (or mixed) distributions assume the local mean, which is the mean of the fast fading distribution, to be lognormally distributed. The best-known composite distributions are Rayleighlognormal, also known as Suzuki, Rice-lognormal, and Nakagami-m-lognormal.

In fact, the Rayleigh distribution constitutes a special case of the Rice, Nakagami-m, Weibull, and of the composite distributions and can be obtained in an exact manner by appropriately setting the parameters of these distributions. Nakagami-m and Rice are found to approximate each other by some simple equations relating the physical parameters associated to each distribution.

Among these, the Nakagami-m distribution has been given a special attention for its ease of manipulation and wide range of applicability [3]. Although, in general, it has been found that the fading statistics of the mobile radio channel may well be characterized by the Nakagami-m, situations are easily found for which other distributions such as Rice and even Weibull yield better results $[4,5]$. More importantly, situations are encountered for which no distributions seem to adequately fit experimental data, though one or another may yield a moderate fitting. Some researches [5] even question the use of the Nakagami-m distribution because its tail does not seem to yield a good fitting to experimental data, better fitting being found around the mean or median.

The well-known fading distributions have been derived assuming a homogeneous diffuse scattering field, resulting from randomly distributed point scatterers. With such an assumption, the central limit theorem leads to complex Gaussian processes with in-phase and quadrature Gaussian distributed variables $x$ and $y$ having zero means and equal standard deviations. In case one cluster of multipath wave is considered then the Rayleigh distribution can be obtained. If a specular component predominates over the scattered 
waves, then the Rice distribution is accomplished. The Nakagami signal can be understood as composed of clusters of multipath waves so that within any one cluster the phases of scattered waves are random and have similar delay times with delay-time spreads of different clusters being relatively large. The assumption of a homogeneous diffuse scattering field is certainly an approximation because the surfaces are spatially correlated characterizing a non-homogeneous environment [1].

This paper presents two general fading distributions - the $\kappa-\mu$ Distribution and the $\eta-\mu$ Distribution. The $\kappa-\mu$ Distribution includes the Rice and the Nakagami-m distributions as special cases. The $\eta-\mu$ Distribution includes the Hoyt and the Nakagami-m distributions as special cases. Therefore, in both fading distributions, the One-Sided Gaussian and the Rayleigh distributions also constitute special cases and the Lognormal distribution may be wellapproximated. Preliminary results show that these new distributions provide a very good fitting to experimental data.

\section{THE $\kappa-\mu$ DISTRIBUTION}

The $\kappa-\mu$ distribution is a general fading distribution that can be used to represent the small scale variation of the fading signal. For a fading signal with envelope $r$ and normalized envelope $\rho=r / \hat{r}, \hat{r}=\sqrt{E\left(r^{2}\right)}$ being the $m s$ value of $r$, the $\kappa-\mu$ probability density function $p(\rho)$ is written as

$$
\begin{aligned}
p(\rho) & =\frac{2 \mu(1+\kappa)^{\frac{\mu+1}{2}}}{\kappa^{\frac{\mu-1}{2}} \exp (\mu \kappa)} \rho^{\mu} \exp \left(-\mu(1+\kappa) \rho^{2}\right) \\
& \times I_{\mu-1}(2 \mu \sqrt{\kappa(1+\kappa) \rho})
\end{aligned}
$$

where $\kappa \geq 0$ is the ratio between the total power of the dominant components and the total power of the scattered waves, $\mu \geq 0$ is given by $\mu=\frac{E^{2}\left(r^{2}\right)}{\operatorname{Var}\left(r^{2}\right)} \times \frac{1+2 \kappa}{(1+\kappa)^{2}}$ (or equivalently, $\left.\mu=\frac{1}{\operatorname{Var}\left(\rho^{2}\right)} \times \frac{1+2 \kappa}{(1+\kappa)^{2}}\right), \quad$ and $I_{v}($.$) is the$ modified Bessel function of the first kind and arbitrary order $V$ ( $V$ real).

For a fading signal with power $w=r^{2} / 2$ and normalized power $\omega=w / \bar{w}$, where $\bar{w}=E(w)$, the $\kappa-\mu$ probability density function $p(\omega)$ is given by

$$
\begin{aligned}
p(\omega)= & \frac{\mu(1+\kappa)^{\frac{\mu+1}{2}}}{\kappa^{\frac{\mu-1}{2}} \exp (\mu \kappa)} \omega^{\frac{\mu-1}{2}} \exp (-\mu(1+\kappa) \omega) \\
& \times I_{\mu-1}(2 \mu \sqrt{\kappa(1+\kappa) \omega})
\end{aligned}
$$

In particular, we may also write $\mu=\frac{E^{2}(w)}{\operatorname{Var}(w)} \times \frac{1+2 \kappa}{(1+\kappa)^{2}}$ (or equivalently $\left.\mu=\frac{1}{\operatorname{Var}(\omega)} \times \frac{1+2 \kappa}{(1+\kappa)^{2}}\right)$.

\subsection{PHYSICAL MODEL: $\kappa \kappa \mu$ DISTRIBUTION}

The fading model for the $\kappa-\mu$ Distribution considers a signal composed of clusters of multipath waves propagating in an non-homogeneous environment. Within any one cluster, the phases of the scattered waves are random and have similar delay times with delay-time spreads of different clusters being relatively large. The clusters of multipath waves are assumed to have the scattered waves with identical powers but within each cluster a dominant component is found that presents an arbitrary power.

\subsection{DERIVATION OF THE $\kappa \mu$ DISTRIBUTION}

Given the physical model for the $\kappa-\mu$ Distribution the envelope, the envelope $r$ can be written in terms of the inphase and quadrature components of the fading signal as

$$
r^{2}=\sum_{i=1}^{n}\left(x_{i}+p_{i}\right)^{2}+\sum_{i=1}^{n}\left(y_{i}+q_{i}\right)^{2}
$$

where $x_{i}$ and $y_{i}$ are mutually independent Gaussian processes with $E\left(x_{i}\right)=E\left(y_{i}\right)=0, E\left(x_{i}^{2}\right)=E\left(y_{i}^{2}\right)=\sigma^{2}$, and $p_{i}$ and $q_{i}$ are respectively the mean values of the inphase and quadrature components of the multipath waves of cluster $i$. Now, we form the processes $\xi_{i}=\left(x_{i}+p_{i}\right)^{2}$ and $\psi_{i}=\left(y_{i}+q_{i}\right)^{2}$, so that

$$
\gamma=r_{i}^{2}=\sum_{i=1}^{n} \xi_{i}+\sum_{i=1}^{n} \psi_{i}
$$

Define $p\left(\xi_{i}\right)$ and $p\left(\psi_{i}\right)$ as the densities of $\xi_{i}$ and $\psi_{i}$, respectively. In such a case

$$
p\left(\lambda_{i}\right)=\frac{1}{\sqrt{2 \pi \lambda_{i}} \sigma} \exp \left(-\frac{\lambda_{i}+s_{i}^{2}}{2 \sigma^{2}}\right) \cosh \left(\frac{\sqrt{\lambda_{i}} s_{i}}{\sigma^{2}}\right)
$$

where $\lambda_{i}=\xi_{i}$ and $s_{i}=p_{i}$ or $\lambda_{i}=\psi_{i}$ and $s_{i}=q_{i}$ The Laplace transform $L\left[p\left(\lambda_{i}\right)\right]$ of $p\left(\lambda_{i}\right)$ is found in an exact manner as [6, page 1026, Eq. 29.3.77]

$$
L\left[p\left(\lambda_{i}\right)\right]=\frac{1}{\sqrt{1+2 \sigma^{2} s}} \exp \left(-\frac{s s_{i}^{2}}{1+2 \sigma^{2} s}\right)
$$

where $s$ is the complex frequency (Laplace variable). Knowing that $\xi_{i}$ and $\psi_{i}, i=1,2, \ldots, n$, are mutually 
independent, the Laplace transform $L[p(\gamma)]$ of $p(\gamma)$ is found as a $2 n$-fold multiplication of $L[p(\gamma)]$. Therefore

$$
L[p(\gamma)]=\frac{1}{\left(1+2 s \sigma^{2}\right)^{2}} \exp \left(-\frac{s \sum_{i=1}^{n}\left(p_{i}^{2}+q_{i}^{2}\right)}{1+2 s \sigma^{2}}\right)
$$

whose inverse is given by [6, page 1026, Eq. 29.3.81]

$$
\begin{aligned}
p(\gamma)= & \frac{1}{2 \sigma^{2}}\left(\frac{\gamma}{\sum_{i=1}^{n}\left(p_{i}^{2}+q_{i}^{2}\right)}\right)^{\frac{n-1}{2}} \exp \left[\frac{\gamma+\sum_{i=1}^{n}\left(p_{i}^{2}+q_{i}^{2}\right)}{2 \sigma^{2}}\right] \\
& \times I_{n-1}\left(\frac{\sqrt{\gamma \sum_{i=1}^{n}\left(p_{i}^{2}+q_{i}^{2}\right)}}{\sigma^{2}}\right)
\end{aligned}
$$

It is not difficult to show that $\hat{r}^{2}=E\left[r^{2}\right\rfloor=2 n \sigma^{2}+\sum_{i=1}^{n} c_{i}^{2}$, where $c_{i}^{2}=p_{i}^{2}+q_{i}^{2}$. In the same way it can be shown that

$$
E\left[r^{4}\right]=4 n \sigma^{4}+4 \sigma^{2} \sum_{i=1}^{n} c_{i}^{2}+\left[2 n \sigma^{2}+\sum_{i=1}^{n} c_{i}^{2}\right]^{2} .
$$

Therefore $\operatorname{Var}\left(r^{2}\right)=4 n \sigma^{4}+4 \sigma^{2} \sum_{i=1}^{n} c_{i}^{2}$.

We define

$$
\kappa=\frac{\sum_{i=1}^{n} c_{i}^{2}}{2 n \sigma^{2}}
$$

Note that $\kappa$ is the ratio between the total power of the dominant components and the total power of the scattered waves. Then

$$
\frac{E^{2}\left(r^{2}\right)}{\operatorname{Var}\left(r^{2}\right)}=n \times \frac{(1+\kappa)^{2}}{(1+2 \kappa)}
$$

Note from (5) that $n$ may be totally expressed in terms of physical parameters such as mean squared value, variance of the power, and the ratio of the total power of the dominant components and the total power of the scattered waves of the fading signal. Note also that whereas these physical parameters are of a continuous nature, $n$ is of a discrete nature. It is plausible to presume that if these parameters are to be obtained by field measurements, their ratios, as defined in (5), will certainly lead to figures that may depart from the exact $n$. Several reasons exist for this. One of them, probably the most meaningful one, is that, although the model proposed here is general, it is in fact an approximate solution to the so-called random phase problem, as are approximate solution to the random phase problem all the other well-known fading models. The limitation of the model can be made less stringent by defining $\mu$ as

$$
\mu=\frac{E^{2}\left(r^{2}\right)}{\operatorname{Var}\left(r^{2}\right)} \times \frac{1+2 \kappa}{(1+\kappa)^{2}}
$$

$\mu$ being the real extension of $n$. Non-integer values of the parameter $\mu$ account for a) non-zero correlation among the clusters of multipath components and b) non-Guassianity of the in-phase and quadrature components of the fading signal. (We note that in derivation of the Nakagami-m model [7], the parameter $n$, which describes the number of "component signals" [7], therefore discrete, is also written in terms of the Nakagami continuous parameter $m$ as $m=n / 2$.) It has been observed experimentally by Nakagami [7] that $\frac{E^{2}\left(r^{2}\right)}{\operatorname{Var}\left(r^{2}\right)} \geq \frac{1}{2}$. Therefore, for the $\kappa-\mu$ Distribution

$$
\frac{\mu(1+\kappa)^{2}}{1+2 \kappa} \geq \frac{1}{2}
$$

with $\kappa \geq 0$ and $\mu \geq 0$. Being of an experimental nature [7], the constraint of Equation 7 does not necessarily need to be observed. In fact, the distribution can be used for $\mu$ assuming any real value $\mu \geq 0$ and $\kappa \geq 0$, as already observed. Using the definitions and the considerations as above and by means of a transformation of variables and a series of algebraic manipulations, the $\kappa-\mu$ probability density function of the envelope can be written from (4) as

$$
\begin{aligned}
\hat{r} p(r)= & \frac{2 \mu(1+\kappa)^{\frac{\mu+1}{2}}}{\kappa^{\frac{\mu-1}{2}} \exp (\mu \kappa)}\left(\frac{r}{\hat{r}}\right)^{\mu} \exp \left(-\mu(1+\kappa)\left(\frac{r}{\hat{r}}\right)^{2}\right) \\
& \times I_{\mu-1}\left(2 \mu \sqrt{\kappa(1+\kappa)} \frac{r}{\hat{r}}\right)
\end{aligned}
$$

In the same way, the probability density function of the power is given as

$$
\begin{aligned}
\bar{w} p(w)= & \frac{\mu(1+\kappa)^{\frac{\mu+1}{2}}}{\kappa^{\frac{\mu-1}{2}} \exp (\mu \kappa)}\left(\frac{w}{\bar{w}}\right)^{\frac{\mu-1}{2}} \exp \left(-\mu(1+\kappa)\left(\frac{w}{\bar{w}}\right)\right) \\
& \times I_{\mu-1}\left(2 \mu \sqrt{\kappa(1+\kappa)\left(\frac{w}{\bar{w}}\right)}\right)
\end{aligned}
$$

Equations (8) and (9) in their normalized forms are respectively given by (1) and (2).

\subsection{THE $\kappa-\mu$ DISTRIBUTION AND THE OTHER FADING DISTRIBUTIONS}

The $\kappa-\mu$ Distribution is a general fading distribution that includes the best known fading distributions, namely Rice and Nakagami-m distributions. Note that both Rice and Nakagami-m include the Rayleigh distribution and the Nakagami-m includes the One-Sided Gaussian. Therefore, 
these distributions can also be obtained from the $\kappa-\mu$ Distribution. The Lognormal distribution may also be well approximated by the $\kappa-\mu$ Distribution.

\subsubsection{RICE AND RAYLEIGH}

The Rice distribution describes a fading signal with one cluster of multipath waves in which one specular component predominates over the scattered waves. Therefore, by setting $\mu=1$ in (1), the $\kappa-\mu$ Distribution reduces to

$$
p(\rho)=\frac{2(1+\kappa)}{\exp (\kappa)} \rho \exp \left(-(1+\kappa) \rho^{2}\right) I_{0}(2 \sqrt{\kappa(1+\kappa)} \rho)
$$

which is the Rice probability density function for the normalized envelope. In this case, the parameter $\kappa$ coincides with the well-known Rice parameter $k$. Now setting $\kappa=0$ in (10) (therefore, $\mu=1$ and $\kappa \rightarrow 0$ in the $\kappa-\mu$ Distribution) the Rayleigh distribution can be obtained in an exact manner. Moreover, for $\kappa=m-1+\sqrt{m(m-1)}$ in (10) (therefore $\mu=1$ and $\kappa=m-1+\sqrt{m(m-1)}$ in the $\kappa-\mu$ Distribution), where $m$ is the Nakagami parameter, the Nakagami-m distribution can be obtained in an approximate manner.

\subsubsection{NAKAGAMI-M, RAYLEIGH, AND ONE-SIDED GAUSSIAN}

The Nakagami-m signal can be understood as composed of clusters of multipath waves with no dominant components within any cluster. Therefore, by setting $\kappa=0$ in the $\kappa-\mu$ Distribution it should be possible to obtain the Nakagami-m distribution. We note, however, that, apart from the case $\mu=1$, which has been explored in the previous subsection, the introduction of $\kappa=0$ in the $\kappa-\mu$ Distribution leads to indeterminacy (zero divided by zero). For small arguments of the Bessel function the relation $I_{\mu-1}(z) \approx(z / 2)^{\mu-1} / \Gamma(\mu)$ holds [6, page 375, Eq. 9.6.7]. Using this in (1), and after some algebraic manipulation,

$$
p(\rho)=\frac{2 \mu^{\mu}(1+\kappa)^{\frac{\mu}{2}}}{\exp (\mu \kappa) \Gamma(\mu)} \rho^{2 \mu-1} \exp \left(-\mu(1+\kappa) \rho^{2}\right)
$$

As $\kappa \rightarrow 0$ (11) reduces to

$$
p(\rho)=\frac{2 \mu^{\mu}}{\Gamma(\mu)} \rho^{2 \mu-1} \exp \left(-\mu \rho^{2}\right)
$$

which is the exact Nakagami-m density function for the normalized envelope. In this case, the parameter $\mu$ coincides with the well-known Nakagami parameter $m$. Now setting $\mu=1$ in (12) (therefore, $\mu=1$ and $\kappa \rightarrow 0$ in the $\kappa-\mu$ Distribution) the Rayleigh distribution can be obtained in an exact manner. In the same way, by setting $\mu=0.5$ in (12) (therefore, $\mu=0.5$ and $\kappa \rightarrow 0$ in the $\kappa-\mu$ Distribution) the One-Sided Gaussian distribution can be obtained in an exact manner. Moreover, for $\mu=(1+k)^{2} /(1+2 k)$ in (12) (therefore $\kappa \rightarrow 0$ and $\mu=(1+k)^{2} /(1+2 k)$ in the $\kappa-\mu$ Distribution), where $k$ is the Rice parameter, the Rice distribution can be obtained in an approximate manner. The Lognormal distribution, given as a function of $m$ in (12) of [7], can also be approximated by the $\kappa-\mu$ Distribution for $e^{-1} \leq \rho \leq e$, and for $\kappa \rightarrow 0$ and $\mu=m$.

\subsection{APPLICATION OF THE $\kappa-\mu$ DISTRIBUTION}

The $\kappa-\mu$ Distribution, as implied in its name, is based on two parameters, $\kappa$ and $\mu$. Its use involves a procedure similar to that of the other distributions, as explained next. From (6), it can be seen that the two parameters $\kappa$ and $\mu$ can be expressed in terms of the ratio between the mean squared value and the variance of the power, which is usually defined as $m$. In other words

$$
m=\frac{\mu(1+\kappa)^{2}}{1+2 \kappa}
$$

For a given $m$, the parameters $\kappa$ and $\mu$ are chosen that yield the best fitting. Note, on the other hand, that, for a given $m$, the parameter $\mu$ shall lie within the range $m$ and 0 , obtained for $\kappa=0$ and $\kappa \rightarrow \infty$, respectively. Therefore, for a given $m$

$$
0 \leq \mu \leq m
$$

The parameter $\mu$ is then chosen within the range of (14). Given that $\mu$ has been chosen, then $\kappa$ must be calculated as

$$
\kappa=\frac{m}{\mu}-1+\sqrt{\frac{m}{\mu}\left(\frac{m}{\mu}-1\right)}
$$

so that the relation as in (13) be kept.

\subsection{SAMPLE EXAMPLES OF THE $\kappa-\mu$ DISTRIBUTION}

This section shows some plots of the $\kappa-\mu$ Distribution. Fig. 1 and Fig. 2, respectively, depict a sample of the various shapes of the $\kappa-\mu$ probability density function $p(\rho)$ and probability distribution function $P(\rho)$ as a function of the normalized envelope $\rho$ for the same Nakagami parameter $m=0.5$. Fig. 3 and Fig. 4 do the same but for $m=0.75$; and Fig. 5 and Fig. 6 , for $m=1.0$; and Fig. 7 and Fig. 8, for $m=1.25$; and Fig. 9 and Fig. 10, for $m=1.5$. 
The plots are illustrated for $\kappa \rightarrow \infty, \kappa=8.47,3.43,1.72$, 0.81 , and $\kappa \rightarrow 0$ (which in decibels correspond to approximately $+\infty, 9.28,5.35,2.36,-0.92$, and $-\infty \mathrm{dB})$. The corresponding values of $\mu$ are respectively:

- $\quad 0,0.1,0.2,0.3,0.4$, and 0.5 , for Fig. 1 and Fig. 2;

- $0,0.15,0.3,0.45,0.6$ and 0.75 , for Fig. 3 and Fig. 4;

- $0,0.2,0.4,0.6,0.8$ and 1, for Fig. 5 and Fig. 6;

- $\quad 0,0.25,0.5,0.75,1$ and 1.25 , for Fig. 7 and Fig. 8;

- $0,0.3,0.6,0.9,1.2$, and 1.5, for Fig. 9 and Fig. 10;

In addition, in Fig. 9 and Fig. 10, the curve for $\kappa=1.37$ (@1.36 dB) was plotted with $\mu=1$.

The curves for which $\kappa \rightarrow 0$ coincide with the Nakagami-m curve, in which case $\mu=m$. The curves for which $\mu=1$ coincide with the Rice curve for which $\kappa=k$.

It can be seen that, although the normalized variance (parameter $m$ ) is kept constant for each Figure, the curves are substantially different from each other. And this is

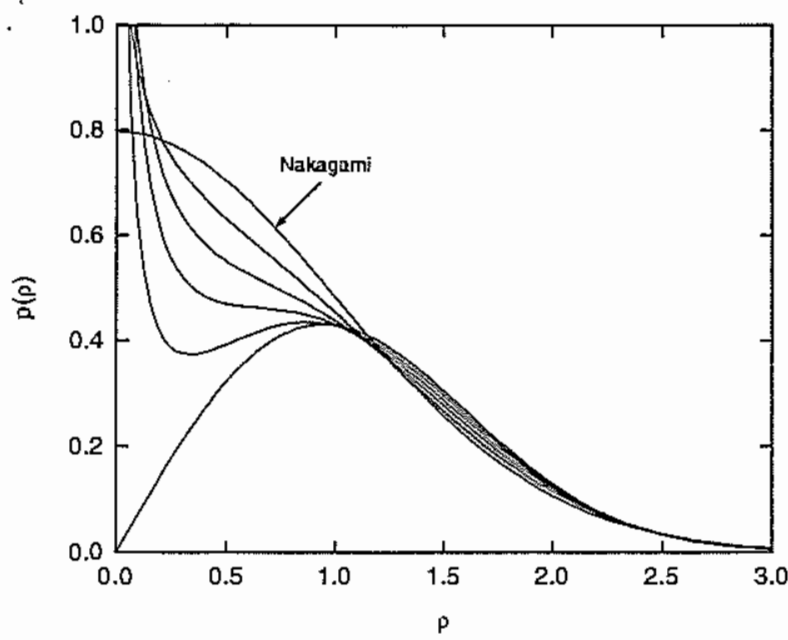

Figure 1. A sample of the various shapes of the $\kappa-\mu$ probability density function for the same Nakagami parameter $m=0.5$. particularly relevant for the distribution function, in which case the lower tail of the distribution may yield differences in the probability of some orders. This feature renders the $\kappa-\mu$ Distribution very flexible and this flexibility can be used in order to adjust the curves to practical data.

\subsection{COMMENTS ON THE $\kappa-\mu$ DISTRIBUTION}

A new general fading distribution - the $\kappa-\mu$ Distribution - has been presented. It models a signal composed of clusters of multipath waves propagating in a nonhomogeneous environment. Within any one cluster, the phases of the scattered waves are random and have similar delay times with delay-time spreads of different clusters being relatively large. The clusters of multipath waves are assumed to have the scattered waves with identical powers but within each cluster a dominant component is found that presents an arbitrary power. The distribution includes the One-Sided Gaussian, the Rayleigh, and, more generally, the Nakagami-m and the Rice distributions as special cases and offers a higher degree of freedom.

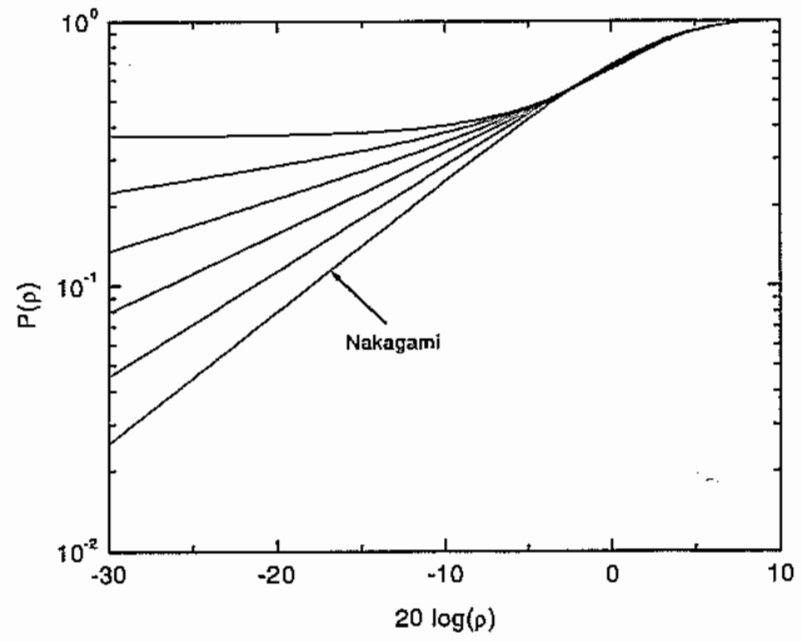

Figure 2. A sample of the various shapes of the $\kappa-\mu$ probability distribution function for the same Nakagami parameter $m=0.5$. 


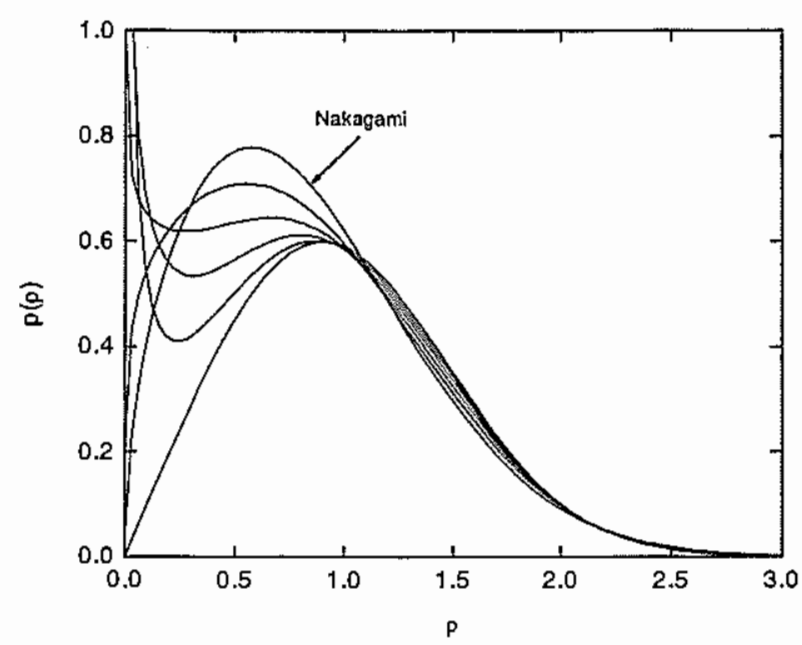

Figure 3. A sample of the various shapes of the $\kappa-\mu$ probability density function for the same Nakagami parameter $m=0.75$.

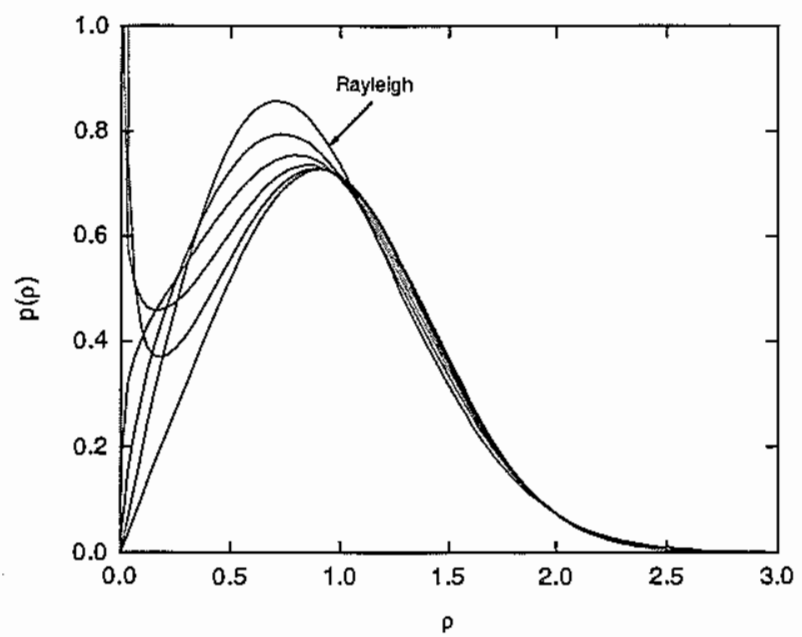

Figure 5. A sample of the various shapes of the $\kappa-\mu$ probability density function for the same Nakagami parameter $m=1.0$.

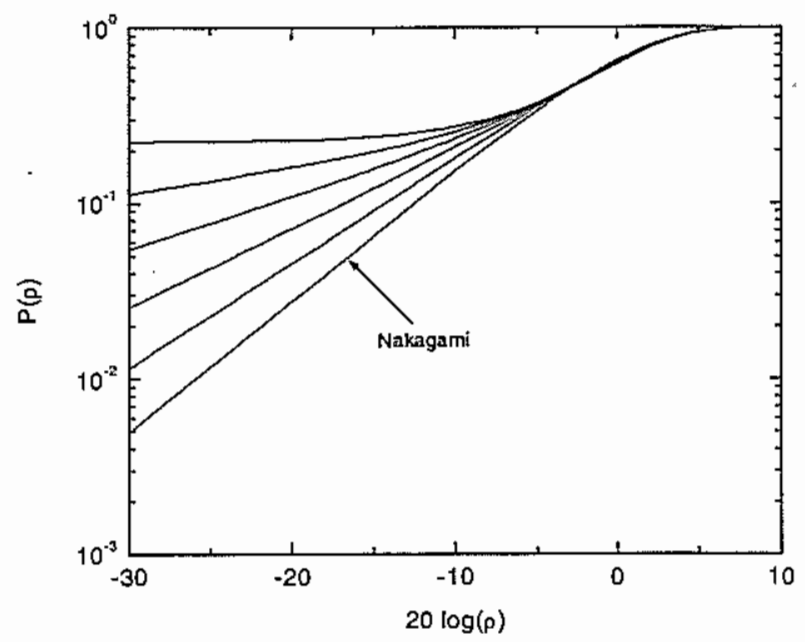

Figure 4. A sample of the various shapes of the $k-\mu$ probability distribution function for the same Nakagami parameter $m=0.75$.

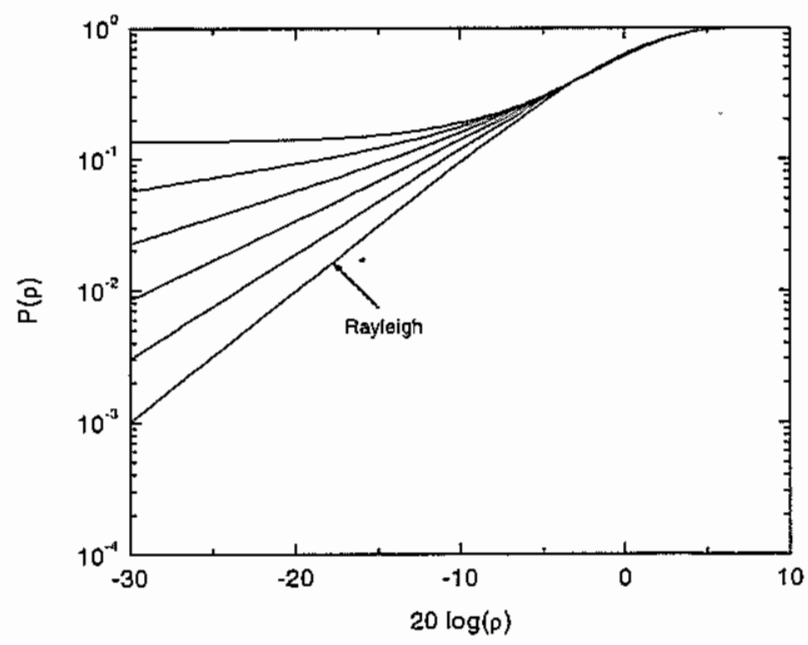

Figure 6. A sample of the various shapes of the $k-\mu$ probability distribution function for the same Nakagami parameter $m=1.0$. 


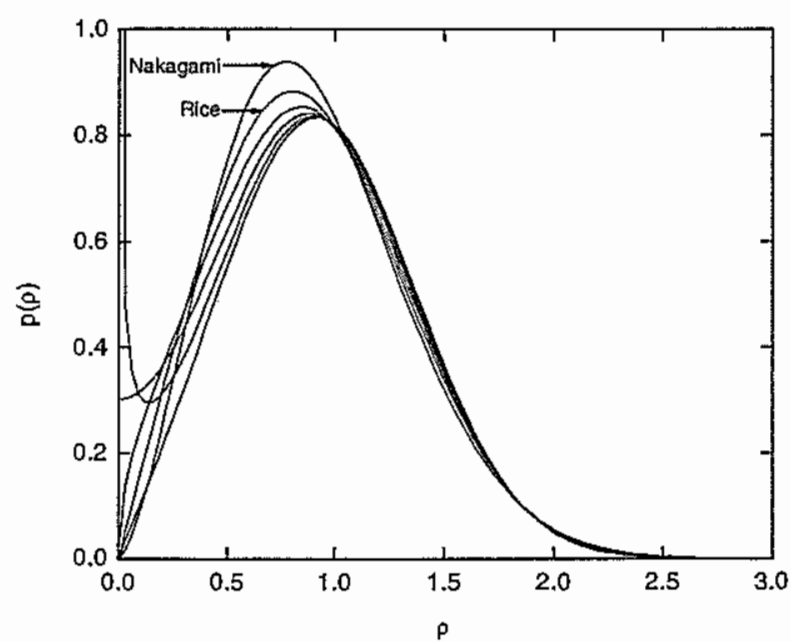

Figure 7. A sample of the various shapes of the $\kappa-\mu$ probability density function for the same Nakagami parameter $m=1.25$.

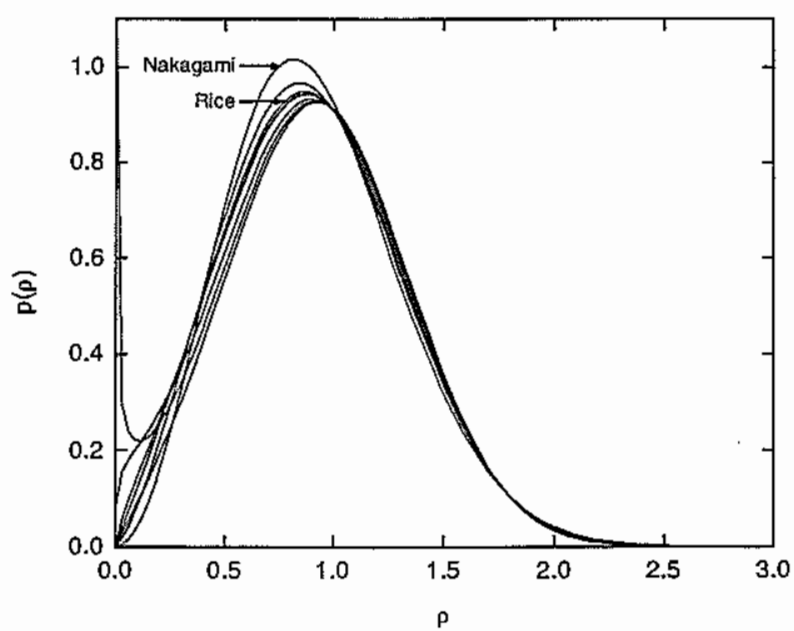

Figure 9. $A$ sample of the various shapes of the $\kappa-\mu$ probability density function for the same Nakagami parameter $m=1.5$.

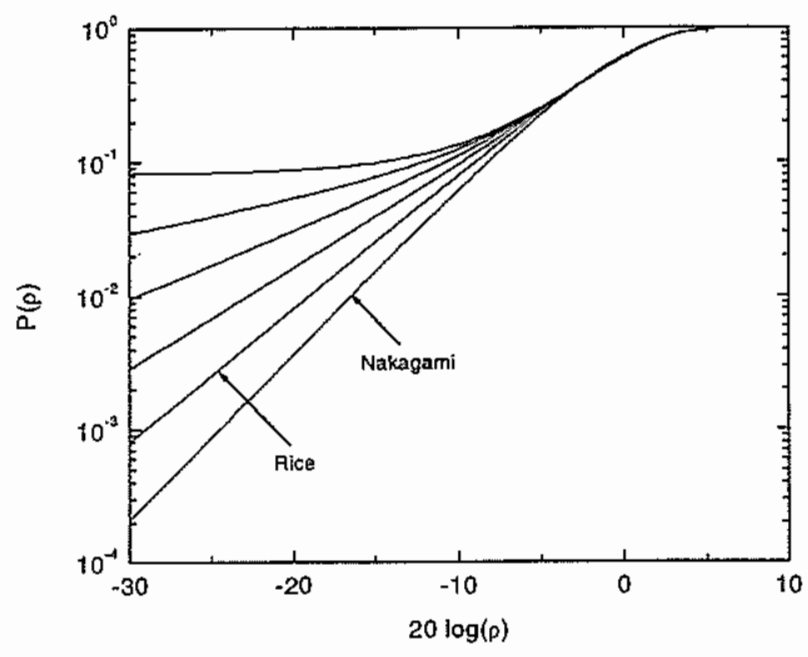

Figure 8. A sample of the various shapes of the $\kappa-\mu$ probability distribution function for the same Nakagami parameter $m=1.25$.

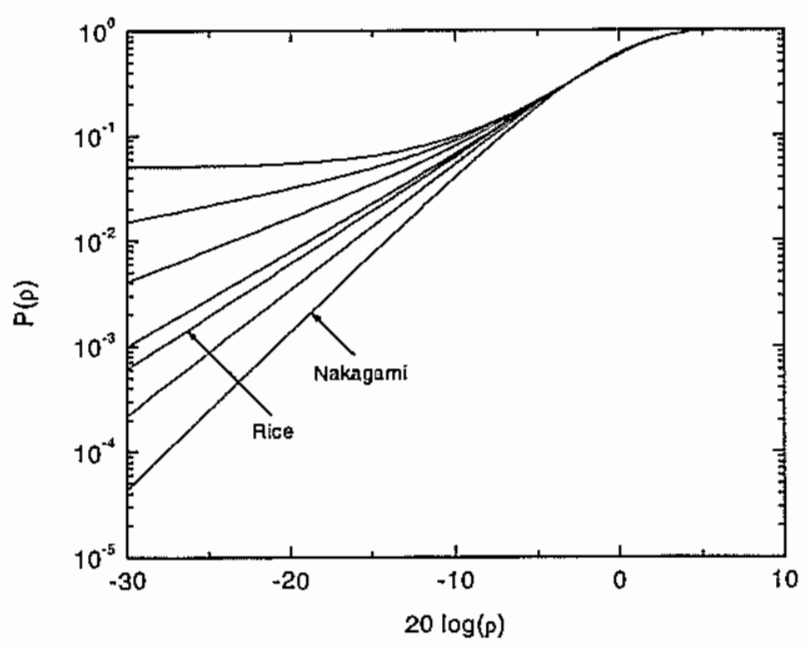

Figure 10. A sample of the various shapes of the $k-\mu$ probability distribution function for the same Nakagami parameter $m=1.5$. 


\section{THE $\eta-\mu$ DISTRIBUTION}

The $\eta-\mu$ distribution is a general fading distribution that can be used to better represent the small scale variation of the fading signal. For a fading signal whose envelope is $r$ and whose envelope $\rho$ normalized with respect to the $m \mathrm{~ms}$ value is given by $\rho=r / \hat{r}, \hat{r}=\sqrt{E\left(r^{2}\right)}$, the $\eta-\mu$ probability density function $p(\rho)$ is written as

$$
\begin{gathered}
p(\rho)=\frac{4 \sqrt{\pi} \mu^{\mu+\frac{1}{2}} h^{\mu}}{\Gamma(\mu) H^{\mu-\frac{1}{2}}} \rho^{2 \mu} \exp \left(-2 \mu h \rho^{2}\right) \\
\quad \times I_{\mu-\frac{1}{2}}\left(2 \mu H \rho^{2}\right)
\end{gathered}
$$

where $h=\frac{2+\eta^{-1}+\eta}{4}, \quad H=\frac{\eta^{-1}-\eta}{4}, \quad \mu=\frac{E^{2}\left(r^{2}\right)}{\operatorname{Var}\left(r^{2}\right)} \times \frac{1+\eta^{2}}{(1+\eta)^{2}}$ (or equivalently, $\left.\mu=\frac{1}{\operatorname{Var}\left(\rho^{2}\right)} \times \frac{1+\eta^{2}}{(1+\eta)^{2}}\right), \quad \Gamma($.$) is the Gamma$ function, $I_{\nu}($.$) is the modified Bessel function of the first$ kind and arbitrary order $v$ ( $v$ real), $\mu \geq 0$ and $0 \leq \eta \leq 1$. (In fact, the distribution is symmetrical for $1 \leq \eta<\infty$, or equivalently $0 \leq \eta^{-1} \leq 1$, in which case $H=\frac{\eta-\eta^{-1}}{4}$. Therefore, more generally, we may write $0 \leq \eta<\infty$ and $H=\left|\eta-n^{-1}\right| / 4$. But, due to the symmetry around 1 , it suffices to consider $\eta$ within one of the ranges only, the range $0 \leq \eta \leq 1$ being preferable for its compactness.)

For a fading signal with power $w=r^{2} / 2$ and normalized power $\omega=w / \bar{w}$, where $\bar{w}=E(w)$, the $\eta-\mu$ probability density function $p(\omega)$ is given by

$$
\begin{aligned}
p(\omega)= & \frac{2 \sqrt{\pi} \mu^{\mu+\frac{1}{2}} h^{\mu}}{\Gamma(\mu) H^{\mu-\frac{1}{2}}} \omega^{\mu-\frac{1}{2}} \exp (-2 \mu h \omega) \\
& \times I_{\mu-\frac{1}{2}}(2 \mu H \omega)
\end{aligned}
$$

In particular, we may also write $\mu=\frac{E^{2}(w)}{\operatorname{Var}(w)} \times \frac{1+\eta^{2}}{(1+\eta)^{2}}$ (or equivalently $\mu=\frac{1}{\operatorname{Var}(\omega)} \times \frac{1+\eta^{2}}{(1+\eta)^{2}}$ ).

\subsection{PHYSICAL MODEL: $\eta-\mu$ DISTRIBUTION}

The fading model for the $\eta-\mu$ Distribution considers a signal composed of clusters of multipath waves propagating in an non-homogeneous environment. Within any one cluster, the phases of the scattered waves are random and have similar delay times with delay-time spreads of different clusters being relatively large. The in-phase and quadrature components of the fading signal within each cluster are assumed to have different powers.

\subsection{DERIVATION OF THE $\eta-\mu$ DISTRIBUTION}

Given the physical model for the $\eta-\mu$ Distribution the envelope $r$ can be written in terms of the in-phase and quadrature components of the fading signal as

$$
r^{2}=\sum_{i=1}^{n}\left(x_{i}^{2}+y_{i}^{2}\right)
$$

where $x_{i}$ and $y_{i}$ are mutually independent Gaussian processes with $E\left(x_{i}\right)=E\left(y_{i}\right)=0, \quad E\left(x_{i}^{2}\right)=\sigma_{x}^{2}$ and $E\left(y_{i}^{2}\right)=\sigma_{y}^{2}$. Now we form the process $r_{i}^{2}=x_{i}^{2}+y_{i}^{2}$, so that $r^{2}=\sum_{i=1}^{n} r_{i}^{2}$. In the same way, we may write $w=\sum_{i=1}^{n} w_{i}$, where $w=r^{2} / 2$ and $w_{i}=r_{i}^{2} / 2$. We proceed to find the density of $r_{i}$. This can be carried by following the standard, but long and tedious, procedure so that

$$
p\left(r_{i}\right)=\frac{\sqrt{\eta} r_{i}}{\sigma_{x}^{2}} \exp \left[-\frac{(1+\eta) r_{i}^{2}}{4 \sigma_{x}^{2}}\right] I_{0}\left[\frac{(\eta-1) r_{i}^{2}}{4 \sigma_{x}^{2}}\right]
$$

where $\eta=\sigma_{x}^{2} / \sigma_{y}^{2}$ and $I_{0}($.$) is the modified Bessel$ function of the first kind order zero. Note that $0 \leq \eta \leq 1$ defines the region within which $\sigma_{x}^{2} \leq \sigma_{y}^{2}$, whereas. $0 \leq \eta^{-1} \leq 1$ defines the region within which $\sigma_{y}^{2} \leq \sigma_{x}^{2}$. It is possible to show that $\hat{r}_{0}^{2} \stackrel{\Delta}{=} E\left(r_{i}^{2}\right)=\left(1+\eta^{-1}\right) \sigma_{x}^{2}$. Therefore

$$
p\left(r_{i}\right)=\frac{2 \sqrt{h} r_{i}}{\hat{r}_{0}^{2}} \exp \left[-h\left(\frac{r_{i}}{\hat{r}_{0}}\right)^{2}\right] I_{0}\left[H\left(\frac{r_{i}}{\hat{r}_{0}}\right)^{2}\right]
$$

where $h$ and $H$ are as previously defined. The density $p\left(w_{i}\right)$ of the power $w_{i}$ is easily found by a simple transformation of variables and it is given by

$$
p\left(w_{i}\right)=\frac{\sqrt{h}}{\bar{w}_{0}} \exp \left(-\frac{h w_{i}}{\bar{w}_{0}}\right) I_{0}\left(\frac{H w_{i}}{\bar{w}_{0}}\right)
$$

where $\bar{w}_{0}=E\left(w_{i}\right)$. The Laplace transform $L\left[p\left(w_{i}\right)\right]$ of $p\left(w_{i}\right)$ is found in an exact manner as [6, pag. 1025, Eq. 29.3.60]

$$
L\left[p\left(w_{i}\right)\right]=\frac{\sqrt{h} / \bar{w}_{0}}{\sqrt{\left(s+h / \bar{w}_{0}\right)^{2}-\left(H / \bar{w}_{0}\right)^{2}}}
$$

Knowing that $w_{i}, i=1,2, \ldots, \mathrm{n}$, are independent, the Laplace transform $L[p(w)]$ of $p(w)$ is found as 


$$
L[p(w)]=\left[\frac{\sqrt{h} / \bar{w}_{0}}{\sqrt{\left(s+h / \bar{w}_{0}\right)^{2}-\left(H / \bar{w}_{0}\right)^{2}}}\right]^{n}
$$

whose inverse is given by [6, pag. 1025, Eq. 29.3.60]

$$
p(w)=\frac{\sqrt{\pi} h^{\frac{n}{2}}}{(2 H)^{\frac{n-1}{2}} \Gamma\left(\frac{n}{2}\right)}\left(\frac{w}{\bar{w}_{0}}\right)^{\frac{n-1}{2}} \exp \left(-\frac{h w}{\bar{w}_{0}}\right) I_{\frac{n-1}{2}}\left(\frac{H w}{\bar{w}_{0}}\right)
$$

We note, however, that $\bar{w}=E(w)=n \bar{w}_{0}$. Therefore

$$
\begin{aligned}
\bar{w} p(w)= & \frac{\sqrt{\pi} n^{\frac{n+1}{2}} h^{\frac{n}{2}}}{(2 H)^{\frac{n-1}{2}} \Gamma\left(\frac{n}{2}\right)}\left(\frac{w}{\bar{w}}\right)^{\frac{n-1}{2}} \exp \left(-\frac{n h w}{\bar{w}}\right) \\
& \times I_{\frac{n-1}{2}}\left(\frac{n H w}{\bar{w}}\right)
\end{aligned}
$$

The corresponding density of the envelope is found to be

$$
\begin{aligned}
\therefore \hat{r} p(r)= & \frac{2 \sqrt{\pi} \frac{\frac{n+1}{2}}{h^{\frac{n}{2}}}}{(2 H)^{\frac{n-1}{2}} \Gamma\left(\frac{n}{2}\right)}\left(\frac{r}{\hat{r}}\right)^{n} \exp \left[-n h\left(\frac{r}{\hat{r}}\right)^{2}\right] \\
& \times \frac{I_{\frac{n-1}{2}}}{2}\left[n H\left(\frac{r}{\hat{r}}\right)^{2}\right]
\end{aligned}
$$

From Equation 18 we find that $E\left(r^{2}\right)=n(1+\eta) \sigma_{y}^{2}$ and $\operatorname{Var}\left(r^{2}\right)=2 n\left(1+\eta^{2}\right) \sigma_{y}^{4}$. Thus

$$
\frac{E^{2}\left(r^{2}\right)}{\operatorname{Var}\left(r^{2}\right)}=\frac{n}{2} \times \frac{(1+\eta)^{2}}{\left(1+\eta^{2}\right)}
$$

Note from Equation 21 that $n / 2$ may be totally expressed in terms of physical parameters such as mean squared value, variance of the power, and power of the inphase and quadrature components of the fading signal. Note also that whereas these physical parameters are of a continuous nature, $n / 2$ is of a discrete nature (integer multiple of $1 / 2$ ). It is plausible to presume that if these parameters are to be obtained by field measurements, their ratios, as defined in Equation 21, will certainly lead to figures that may depart from the exact $n / 2$. Several reasons exist for this. One of them, probably the most significant one, is that, although the model proposed here is general, it is in fact an approximate solution to the so-called random phase problem, as are approximate solution to the random phase problem all the other well-known fading models. The limitation of the model can be made less stringent by defining $\mu$ as

$$
\mu=\frac{E^{2}\left(r^{2}\right)}{\operatorname{Var}\left(r^{2}\right)} \times \frac{1+\eta^{2}}{(1+\eta)^{2}}
$$

$\mu$ being the real extension of $n / 2$. Values of $\mu$ that differ from multiples of $1 / 2$ account for a) non-zero correlation among the clusters of multipath components and b) non-Guassianity of the in-phase and quadrature components of the fading signal. (We note that in derivation of the Nakagami model [7], the parameter $n$, which describes the number of "component signals", therefore discrete, is also written in terms of the Nakagami continuous parameter $m$ as $m=n / 2$.) It has been observed experimentally by Nakagami [7] that

$$
\frac{E^{2}\left(r^{2}\right)}{\operatorname{Var}\left(r^{2}\right)} \geq \frac{1}{2}
$$

Therefore, for the $\eta-\mu$ Distribution

$$
\frac{\mu(1+\eta)^{2}}{I+\eta^{2}} \geq \frac{1}{2}
$$

with

$$
0 \leq \eta \leq 1
$$

(or equivalently $0 \leq \eta^{-1} \leq 1$ ). Being of an experimental nature [7], the constraint of Equation 23 does not necessarily need to be observed. In fact, the distribution can be used for $\mu$ assuming any real value $\mu \geq 0$ and $0 \leq \eta \leq 1$, as already observed.

The probability density function of the envelope can be written as

$$
\begin{gathered}
\hat{r} p(r)=\frac{4 \sqrt{\pi} \mu^{\mu+\frac{1}{2}} h^{\mu}}{\Gamma(\mu) H^{\mu-\frac{1}{2}}}\left(\frac{r}{\hat{r}}\right)^{2 \mu} \exp \left[-2 \mu h\left(\frac{r}{\hat{r}}\right)^{2}\right] \\
\times I_{\mu-\frac{1}{2}}\left[2 \mu H\left(\frac{r}{\hat{r}}\right)^{2}\right]
\end{gathered}
$$

In the same way, the probability density function of the power is obtained as

$$
\begin{aligned}
\bar{w} p(w)= & \frac{2 \sqrt{\pi} \mu^{\mu+\frac{1}{2}} h^{\mu}}{\Gamma(\mu) H^{\mu-\frac{1}{2}}}\left(\frac{w}{\bar{w}}\right)^{\mu-\frac{1}{2}} \exp \left(-\frac{2 \mu h w}{\bar{w}}\right) \\
& \times I_{\mu-\frac{1}{2}}\left(\frac{2 \mu H w}{\bar{w}}\right)
\end{aligned}
$$

which, in the normalized form, are given as in Equations 16 and 17 , respectively. 


\subsection{THE $\eta-\mu$ DISTRIBUTION AND THE OTHER FADING DISTRIBUTIONS}

The $\eta-\mu$ Distribution is a general fading distribution that includes the Hoyt, the One-Sided Gaussian, the Rayleigh, and, more generally, the Nakagami distributions as special cases. Rice and Lognormal distributions may also be wellapproximated by the $\eta-\mu$ Distribution. We note that the One-Side Gaussian and the Rayleigh distributions can be obtained from the Nakagami distribution by setting the Nakagami parameter $m=0.5$ and $m=1$, respectively. Therefore, in order to relate the $\eta$ - $\mu$ Distribution with these two distributions it suffices to relate it with the Nakagami one.

\subsubsection{HOYT, ONE-SIDED, AND RAYLEYGH}

The Hoyt distribution can be obtained from the $\eta-\mu$ Distribution in an exact manner by setting $\mu=1 / 2$. From the Hoyt distribution the One-Sided Gaussian is obtained when $\eta \rightarrow 0$. In the same way, from the Hoyt distribution the Rayleigh distribution is obtained when $\eta=1$.

\subsubsection{NAKAGAMI-M, RAYLEIGH, AND ONE-SIDED GAUSSIAN}

The Nakagami distribution can be obtained in an exact manner from the $\eta-\mu$ Distribution for $\mu=m$ and $\eta \rightarrow 0$ (or equivalently $\eta \rightarrow \infty$ ) or, in the same way, for $\mu=m / 2$ and $\eta \rightarrow 1$. This result is not straightforwardly seen from the densities here derived. We observe, nonetheless, that for these conditions all the Gaussian variates present identical variances and the fading model proposed here deteriorates into that of [8], where the Nakagami distribution is obtained in an exact manner. For intermediate values of $\eta$ the $\eta-\mu$ distribution and the Nakagami distribution relate to each other for $\frac{\mu(1+\eta)^{2}}{1+\eta^{2}}=m$. This is a very interesting result which shows that an infinite number of curves of the $\eta-\mu$ distribution can be found that presents the same Nakagami parameter $m$, conditioned on the fact that the constraints $m / 2 \leq \mu \leq m$ and $\eta=\frac{\frac{\mu}{m}-\sqrt{2 \frac{\mu}{m}-1}}{1-\frac{\mu}{m}}$ are satisfied. The Lognormal distribution, given as a function of $m$ in Equation 13 of [7], can also be approximated by the $\eta-\mu$ Distribution for $e^{-1} \leq \rho \leq e$, and for $\eta, \mu$, and $m$ satisfying the relations given above for the Nakagami case. In the same way, an infinite number of curves of the $\eta-\mu$ Distribution can be found that presents the same Nakagami parameter for the Lognormal distribution. The Rice distribution can be approximated by the $\eta-\mu$ distribution for $\frac{\mu(1+\eta)^{2}}{1+\eta^{2}}=\frac{(1+k)^{2}}{1+2 k}$, where $k \geq 0$ is the Rice parameter. In the same way, this result shows that an infinite number of curves of the $\eta-\mu$ Distribution can be found that presents the same Rice parameter $k$, conditioned on the fact that the constraints

$$
\frac{(1+k)^{2} / 2}{2 k+1} \leq \mu \leq \frac{(1+k)^{2}}{2 k+1}
$$

and

$$
\eta=\left(\frac{\mu(1+2 k)}{(1+k)^{2}}-\sqrt{\frac{2 \mu(1+2 k)}{(1+k)^{2}}-1}\right) /\left(1-\frac{\mu(1+2 k)}{(1+k)^{2}}\right)
$$

are satisfied.

\subsection{SAMPLE EXAMPLES OF THE $\eta-\mu$ DISTRIBUTION}

This section shows some plots of the $\eta-\mu$ Distribution. Fig. 11 and Fig. 12, respectively, depict a sample of the various shapes of the $\eta$ - $\mu$ probability density function $p(\rho)$ and probability distribution function $P(\rho)$ as a function of the normalized envelope $\rho$ for the same Nakagami parameter $m=0.5$. Fig. 13 and Fig. 14 do the same but for $m=0.75$; and Fig. 15 and Fig. 16 , for $m=1.0$; and Fig. 17 and Fig. 18, for $m=1.25$; and Fig. 19 and 20, for $m=1.5$. The plots are illustrated for $\eta \rightarrow 0, \eta=0.005$, $0.026,0.055,0.127,0.225,0.382$, and $\eta \rightarrow 1$ (which in decibels correspond to approximately $-\infty,-23.01,-15.85$, $12.60,-8.96,-6.48,-4.18$ and $0 \mathrm{~dB}$ ). The corresponding values of $\mu$ are respectively:

- $\quad 0.5,0.495,0.475,0.45,0.4,0.35,0.3,0.25$, for Fig. 11 and Fig. 12;

- $\quad 0.75,0.7425,0.7125,0.675,0.6,0.525,0.45,0.375$, for Fig. 13 and Fig. 14;

- $1.0,0.99,0.95,0.9,0.8,0.7,0.6,0.5$, for Fig. 15 and Fig. 16;

- $1.25,1.2375,1.1875,1.125,1.0,0.875,0.75,0.625$, for Fig. 17 and Fig. 18;

- $\quad 1.5,1.485,1.425,1.35,1.2,1.05,0.9,0.75$, for Fig. 19 and 20 ;

The curves for which $\eta \rightarrow 1$ and $\eta \rightarrow 0$ coincide with each other and also with the Nakagami one, as indicated in the Figures. In such cases, we have $\mu=m / 2$ and $\mu=m$, respectively. It can be seen that, although the normalized variance (parameter $m$ ) is kept constant for each Figure, the curves are substantially different from each other. And this is particularly noticeable for the distribution function, in which case the lower tail of the distribution may yield differences in the probability of some orders. Moreover, the curves present a very interesting feature, as described next. For the same value of $m$ and departing from the condition for which $\eta \rightarrow 1$, as $\eta$ diminishes the curves depart from that for which $\eta \rightarrow 1$, initially keeping a similar shape. As $\eta$ diminishes the shapes of the curves change substantially.

As $\eta$ diminishes even further and as $\eta \rightarrow 0$, the curves merge with that of the initial shape but such curves present shapes very different from those obtained as $\eta \rightarrow 0$. This feature renders the $\eta-\mu$ Distribution very flexible and this flexibility can be used in order to adjust the curves to practical data. 


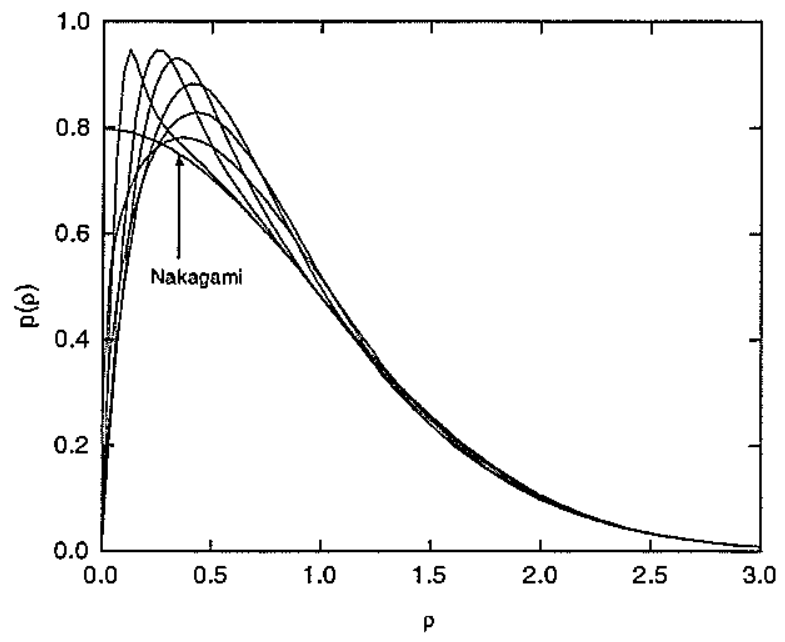

Figure 11. A sample of the various shapes of the $\eta-\mu$ probability density function for the same Nakagami parameter $m=0.5$.

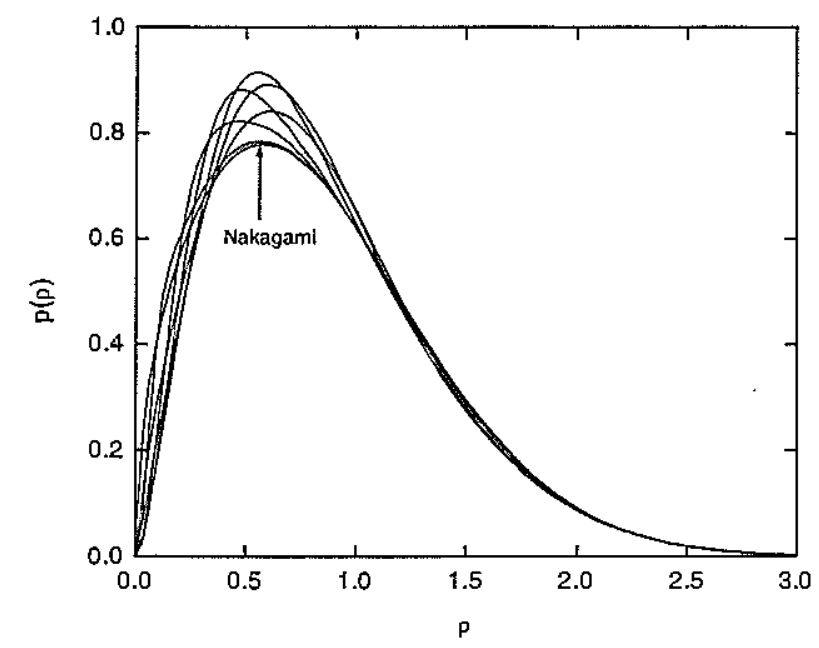

Figure 13. A sample of the various shapes of the $\eta-\mu$ probability density function for the same Nakagami parameter $m=0.75$.

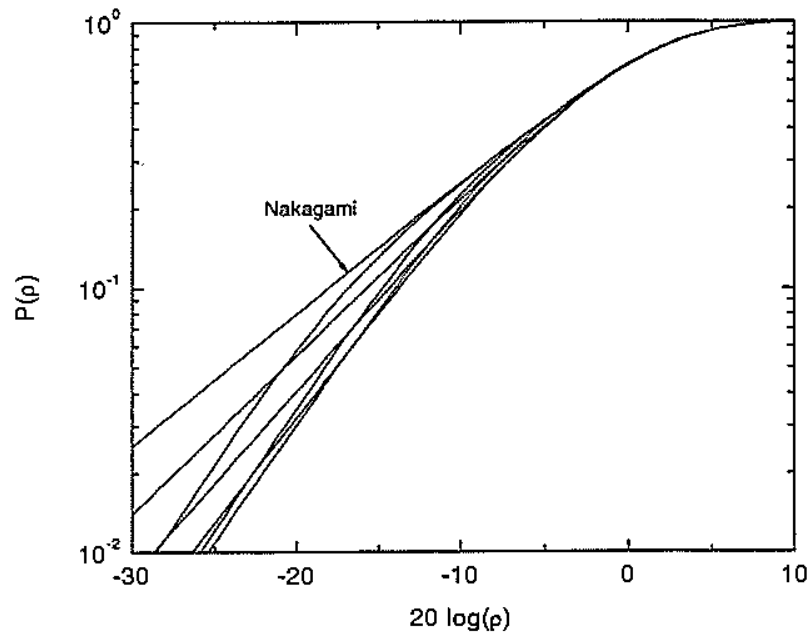

Figure 12. A sample of the various shapes of the $\eta-\mu$ probability distribution function for the same Nakagami parameter $m=0.5$.

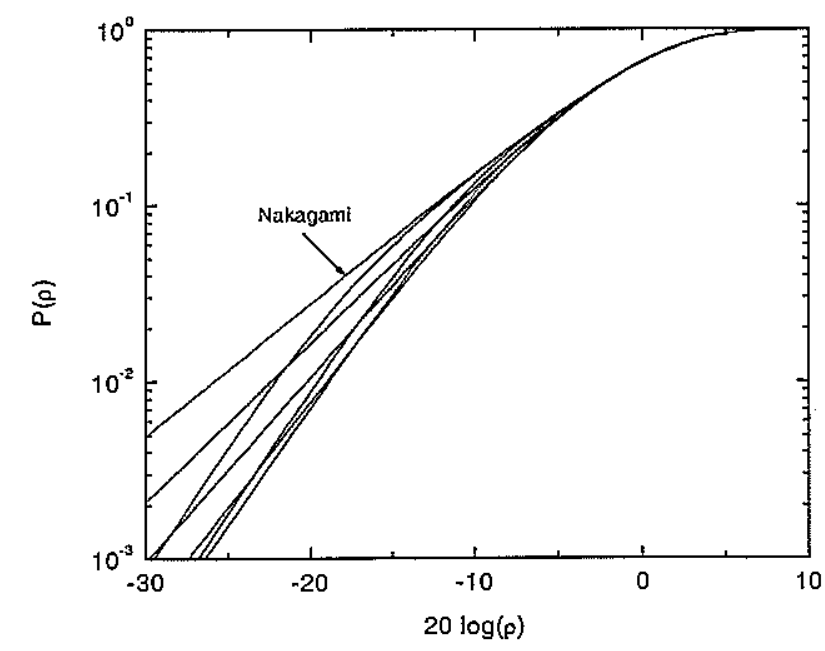

Figure 14. A sample of the various shapes of the $\eta-\mu$ probability distribution function for the same Nakagami parameter $m=0.75$. 


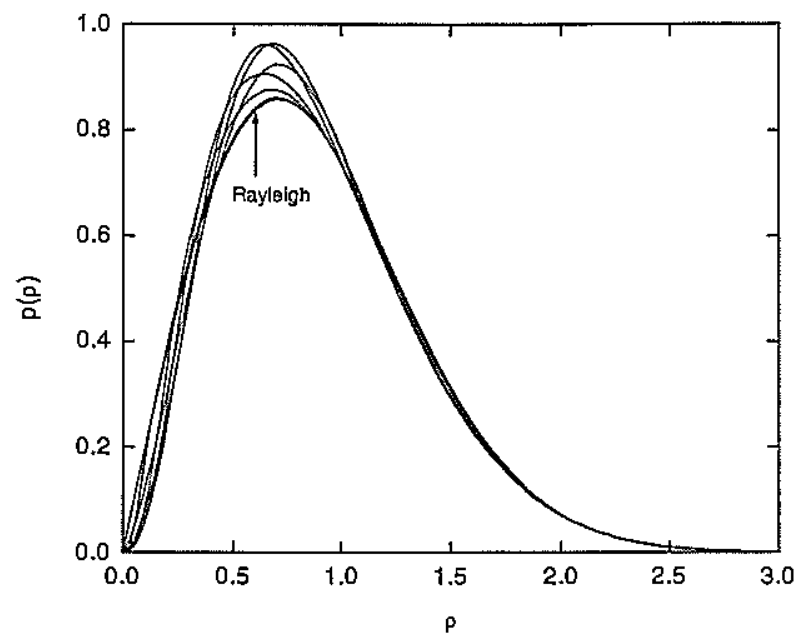

Figure 15. A sample of the various shapes of the $\eta-\mu$ probability density function for the same Nakagami parameter $m=1.0$.

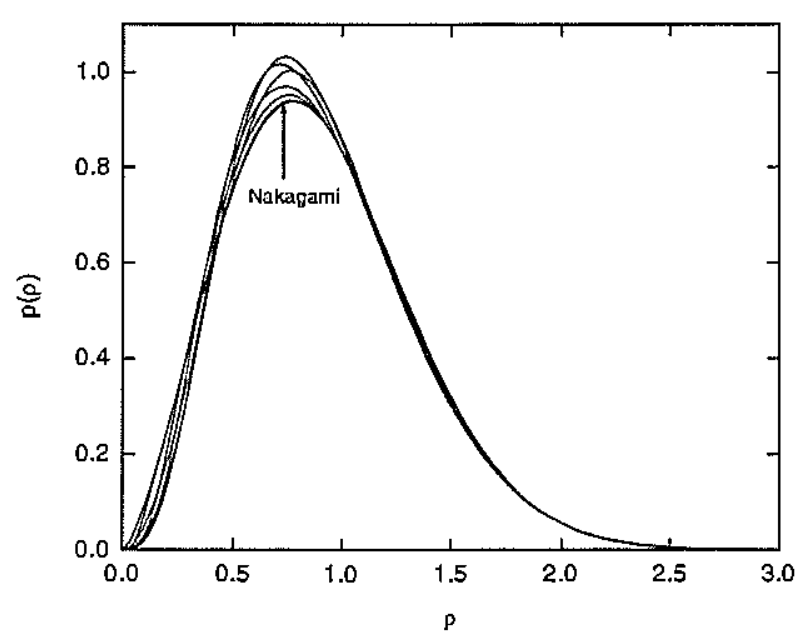

Figure 17. A sample of the various shapes of the $\eta-\mu$ probability density function for the same Nakagami parameter $m=1.25$.

\subsection{COMMENTS ON THE $\eta-\mu$ DISTRIBUTION}

A new general fading distribution - the $\eta-\mu$ Distribution - has been presented. It models a signal composed of clusters of multipath waves propagating in a nonhomogeneous environment. Within any one cluster, the phases of the scattered waves are random and have similar

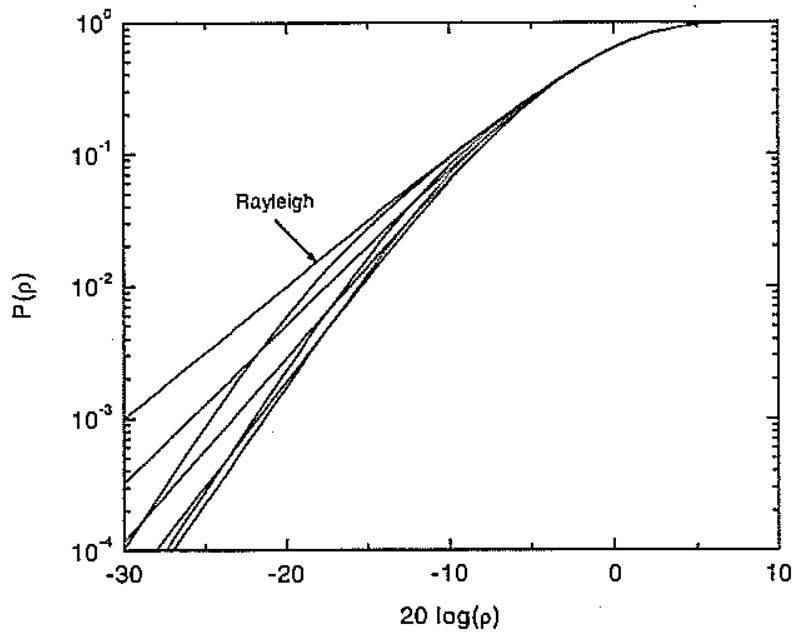

Figure 16. A sample of the various shapes of the $\eta-\mu$ probability distribution function for the same Nakagami parameter $m=1.0$.

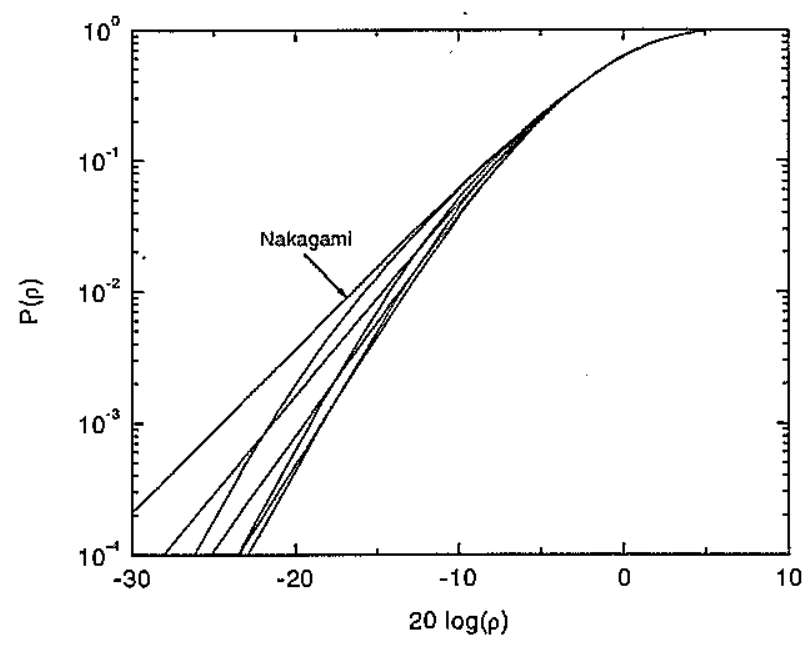

Figure 18. A sample of the various shapes of the $\eta-\mu$ probability distribution function for the same Nakagami parameter $m=1.25$.

delay times with delay-time spreads of different clusters being relatively large. The clusters of multipath waves are assumed to have the scattered waves with different powers and no dominant component is found. The distribution includes the One-Sided Gaussian, the Rayleigh, the Hoyt and, more generally, the Nakagami-m distributions as special cases and offers a higher degree of freedom. 


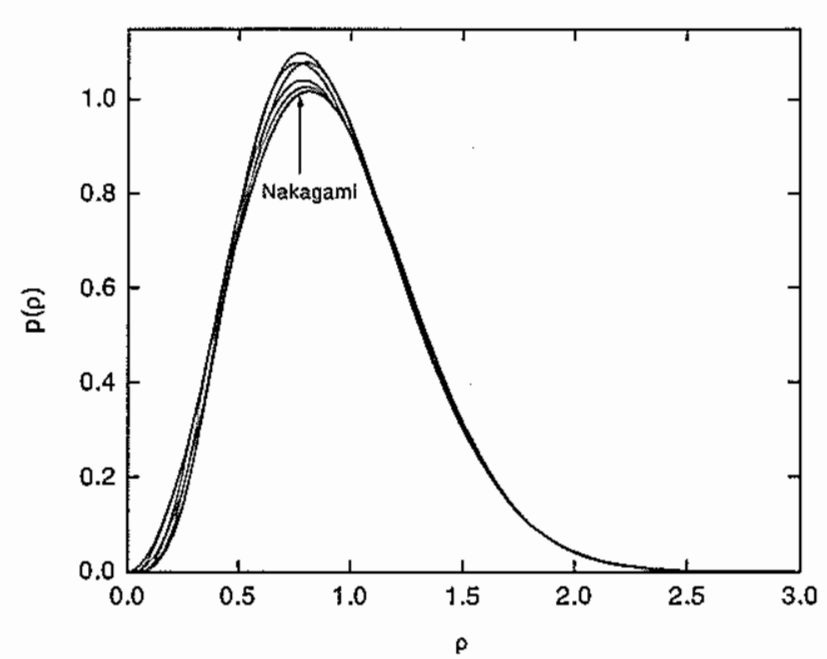

Figure 19. A sample of the various shapes of the $\eta-\mu$ probability density function for the same Nakagami parameter $m=1.5$.

\section{CONCLUSIONS}

This paper presented two general fading distributions the $\kappa-\mu$ Distribution and the $\eta-\mu$ Distribution. The $\kappa-\mu$ Distribution includes the Rice and the Nakagami-m distributions as special cases. The $\eta-\mu$ Distribution includes the Hoyt and the Nakagami-m distributions as special cases. Further investigations, not included here, show that the Nakagami distribution can be thought of as a mean distribution which divides the fading plane into two: the upper plane is then described by the $\kappa-\mu$ Distribution whereas the lower plane is described by the $\eta-\mu$ Distribution. Because these distributions are more flexible than the other fading distributions they can yield better fitting to experimental data.

\section{ACKNOWLEDGEMENTS}

The author is thankful to Álvaro Augusto Machado de Medeiros, Gustavo Frainderaich, José Cândido Silveira Santos Filho, and Maurício Sol de Castro for their help.

\section{REFERENCES}

[1] W. R. Braun and U. Dersch, "A physical mobile radio channel model," IEEE Trans. Veh. Technol. vol. 40, no. 2, pp. 472482, May 1991.

[2] G. L. Turin, "Introduction to spread-spectrum antimultipath techniques and their application to urban digital radio," Proc. IEEE, vol. 68, no. 3, pp. 328-353, March 1980.

[3] H. Suzuki, "A statistical model for urban radio propagation," IEEE Trans. Commun., vol. COM-25, no. 7, pp. 673-679, July 1977.

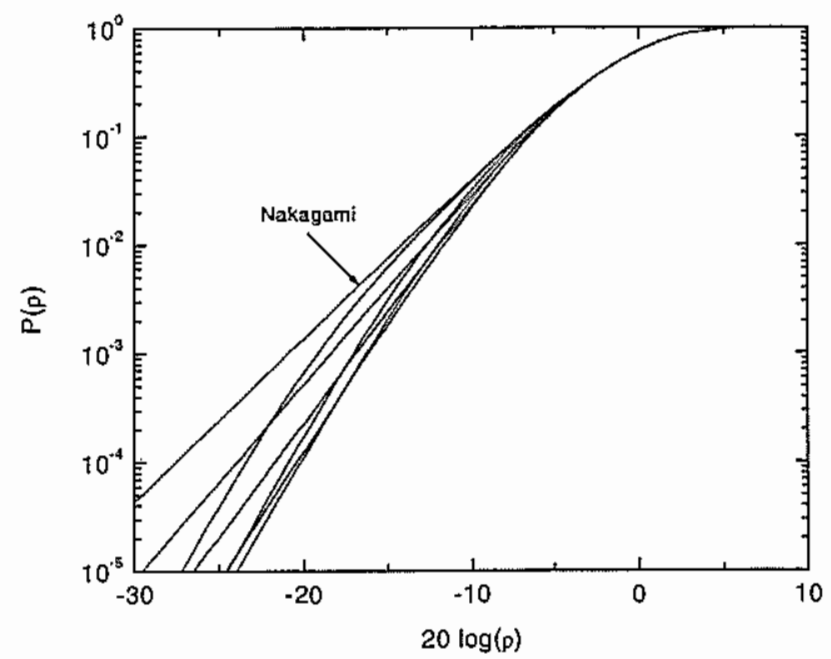

Figure 20. A sample of the various shapes of the $\eta-\mu$ probability distribution function for the same Nakagami parameter $m=1.5$.

[4] J. D. Parsons, The Mobile Radio Channel, New York: Halsted Press, 1992.

[5] S. Stein, "Fading channel issues in system engineering," IEEE J. Selected Areas in Commun., vol. 5, no. 2 , pp. 68-69, Feb. 1987.

[6] M. Abramowitz, I. A. Stegun, Hanbook of Mathematical Functions, US Dept. of Commerce, National Bureau of Standards, Applied Mathematics Series, 1972.

[7] M. Nakagami, "The m-distribution - A general formula of intensity distribution of rapid fading, in Statistical Methods in Radio Wave Propagation," W. C. Hoffman, Ed. Elmsford, NY: Pergamon, 1960.

[8] M. D. Yacoub, J. E. Vargas B., L. G. de R. Guedes, "On higher order statistics of the Nakagami-m distribution," IEEE Trans. Veh. Technol., vol. 48, no. 3, pp. 790-794, May 1999.

Michel Daoud Yacoub received the B.S.E.E. and the M.Sc degrees from the School of Electrical and Computer Engineering of the State University of Campinas, UNICAMP, Brazil, respectively in 1978 and 1983, and the PhD. degree from the University of Essex, England, in 1988. From 1978 to 1985, he worked as a research specialist at the R\&D Center of Telebrás, Brazil, in the development of the Tropico digital exchange family. $\mathrm{He}$ joined the School of Electrical and Computer Engineering, UNICAMP, in 1989, where he is presently a Full Professor. He consults for several operating companies and industries in the wireless communications area. $\mathrm{He}$ is the author of the books Foundations of Mobile Radio Engineering, CRC Press, 1993, Wireless Technology: Protocols, Standards, and Techniques, CRC Press, 2001, and the co-author of the book Telecommunications: Principles and Trends (in Portuguese), Erica Press, 1997. He holds two patents. His research interests include wireless commumications in general. 